\title{
A 14-month study of change in disability and mood state in patients with chronic migraine associated to medication overuse
}

\author{
A. Raggi • M. Leonardi - A. M. Giovannetti • \\ S. Schiavolin • G. Bussone $\cdot$ L. Grazzi $\cdot$ \\ S. Usai · M. Curone $\cdot$ P. Di Fiore $\cdot$ D. D'Amico
}

(C) Springer-Verlag Italia 2013

\begin{abstract}
This paper aims to evaluate changes in disease severity, disability and mood state in patients with chronic migraine associated to medication overuse (CM-MO). MIDAS was used for assessing disease activity, WHO-DAS2 for disability, DBI-2 for mood state. ANOVA was used to test change over time; $t$-test to assess follow-up differences in WHO-DAS- 2 and BDI- 2 between patients with MIDAS $\leq 20$ and $\geq 21$. Change in MIDAS, WHO-DAS-2 and BDI-2 scores were computed: Pearson's index was used to assess correlation between them; linear regression to assess change in WHO-DAS-2, using MIDAS and BDI-2 change as predictors. Mean MIDAS decreased significantly (from 101.9 to 52.0). In $26.1 \%$ of the sample, MIDAS fell below 21 at follow-up: these patients had lower WHO-DAS-2 score. WHO-DAS- 2 change was little correlated to MIDAS change and strongly correlated to changes in BDI-2 scores. $57.1 \%$ of WHO-DAS-2 change variance is explained by change in BDI-2 and MIDAS scores. There was a clear clinical improvement 14 months after detoxification, and a modest reduction in disability which is explained by reduced disease activity and improved mood state. An appropriate treatment of CM-MO, based on detoxification and prophylaxis, is likely to reduce disease burden: recognition and treatment of mood problems may be a key factor to reduce disability.
\end{abstract}

\footnotetext{
A. Raggi $(\varangle) \cdot$ M. Leonardi · A. M. Giovannetti · S. Schiavolin Neurology Public Health and Disability Unit, Neurological Institute C. Besta IRCCS Foundation, Via Celoria 11, 20133 Milan, Italy

e-mail: araggi@istituto-besta.it

G. Bussone · L. Grazzi - S. Usai · M. Curone · P. Di Fiore ·

D. D'Amico

Department of Clinical Neuroscience, Headache Unit,

Neurological Institute C. Besta IRCCS Foundation,

Via Celoria 11, 20133 Milan, Italy
}

Keywords Chronic Migraine - Disability · WHO-DAS-2 . Mood · Prophylaxis

\section{Introduction}

Chronic migraine associated to medication overuse (CM$\mathrm{MO}$ ) is an unfavourable outcome of migraine course, which seems to be mediated by lifestyle, life events, comorbid conditions, genetic terrain and medication overuse [1, 2], and which determines a relevant burden, in particular in terms of increased disease cost [3]. A recent review showed that patients with a chronic form of headache report a remarkable impact on functioning, with high disability and reduction in work and non-work activities [4]. Compared to those with episodic migraine (EM), CM-MO patients show increased disability and reduced mood level [5], and are twice likely to have depression [6]. Therapeutic approach requires withdrawal, followed by prophylactic intervention [7]: this approach is effective in reducing the frequency of headaches and patients' disability up to 5 years after detoxification [8], but it is not clear to what extent mood state and disease severity change may impact over patients' disability. The aim of this paper was to longitudinally assess the impact of disease activity and mood state change over disability change in a group of CM-MO patients.

\section{Methods}

In this longitudinal study, patients with CM-MO according to Silberstein's criteria [9] were consecutively enrolled and followed up for 12-18 months. Disease activity was assessed with the Migraine Disability Assessment (MIDAS) [10], disability with the WHO Disability Assessment 
Table 1 Linear regression: BDI-2 and MIDAS changes as predictors of WHO-DAS-2 change

\begin{tabular}{|c|c|c|c|c|}
\hline Model & $\begin{array}{l}R \\
0.768\end{array}$ & $\begin{array}{l}R^{2} \\
0.590\end{array}$ & $\begin{array}{l}\text { Adj. } R^{2} \\
0.571\end{array}$ & $\begin{array}{l}\mathrm{F} \\
31.22 \\
t\end{array}$ \\
\hline Coefficients & $B$ & Std err & $\beta$ & $t$ \\
\hline BDI-2 somatic & 1.092 & 0.267 & 0.481 & $4.10 * *$ \\
\hline BDI-2 cognitive & 0.979 & 0.385 & 0.302 & $2.54 *$ \\
\hline MIDAS & 0.024 & 0.016 & 0.123 & 1.50 \\
\hline
\end{tabular}

$* P<0.05, * * P<0.01$

Schedule-2 (WHO-DAS-2) [11], mood state with the Beck Depression Inventory-2 (BDI-2) [12].

Longitudinal differences were tested with ANOVA; differences in WHO-DAS-2 and BDI-2 between those with MIDAS $\leq 20$ (i.e. below high severity threshold) and $\geq 21$ at follow-up were assessed with $t$-test. Changes in MIDAS, WHO-DAS-2 and BDI-2 scores were computed, and correlations between each other were calculated with Pearson's index. Linear regression was used to assess change in WHO-DAS-2, using MIDAS and BDI-2 changes as predictors. All analyses were carried out on study completers only, $P$ value $<0.05$ was used to set statistical significance.

\section{Results}

In total, 69 patients (56 females, mean age 42.2, SD 12.6) completed follow-up: mean follow-up duration was 14 months (SD 1.7). Longitudinal differences were significant for MIDAS score only $(101.9 \pm 79.3$ at baseline, $52.0 \pm 54.3$ at follow-up, $P<0.001$ ), for the remaining variables a trend of improvement was observed (i.e. a decrease in WHO-DAS-2 and BDI-2 scores), but no statistical significance was detected. MIDAS fell below 21 in $26.15 \%$ of the sample: compared to the other patients, these reported significantly lower WHO-DAS-2 (14.4 \pm 13.4 Vs. $30.8 \pm$ $18.3, P<0.05)$, while no difference was observed at BDI-2.

WHO-DAS-2 change was little correlated to MIDAS change $(R=0.28, P<0.05)$ and strongly correlated to changes in BDI-2 Somatic $(R=0.73, P<0.001)$, Cognitive $(R=0.69, P<0.001)$ and Summary scores $(R=0.76$, $P<0.001)$. Table 1 shows the regression analysis in which MIDAS and BDI-2 change predicted WHO-DAS-2 change. The model explained $57.1 \%$ of variation: BDI-2 Somatic and Cognitive were independent predictors.

\section{Discussion}

Our data show a clear clinical improvement 14 months after detoxification: although only one-fourth of patients achieved a MIDAS score $\leq 21$, mean MIDAS reduction was around $50 \%$, while disability and mood state improvement were modest. Improvement in disability is largely explained by reduced disease activity and, in a much more significant way, by improved mood state. Our findings therefore indicate that an appropriate treatment of CM-MO, based on detoxification and prophylaxis, is likely to reduce disease's burden. Moreover, our findings suggest that recognition and treatment of mood problems may be a key factor to promote a reduction in disability. Our results support previous findings showing that depression is associated to a higher risk of developing CM [13], and that this condition is more frequent in CM-MO patients compared to EM patients [14].

Our results are limited by small sample size and by the fact that the analyses were based on study completers only; therefore, these have to be intended as preliminary indications only.

Conflict of interest The authors certify that there is no actual or potential conflict of interest in relation to this article.

\section{References}

1. Bigal ME, Lipton RB (2011) Migraine chronification. Curr Neurol Neurosci Rep 11:139-148

2. Bigal ME, Serrano D, Buse D et al (2008) Acute migraine medications and evolution from episodic to chronic migraine: a longitudinal population-based study. Headache 48:1157-1168

3. Linde M, Gustavsson A, Stovner LJ et al (2012) The cost of headache disorders in Europe: the Euro light project. Eur J Neurol 19:703-711

4. D'Amico D, Grazzi L, Usai S et al (2011) Disability in chronic daily headache: state of the art and future directions. Neurol Sci 32:S71-S76

5. Raggi A, Giovannetti AM, Leonardi M et al (2012) Disability and mood state in patients with episodic and chronic migraine associated to medication overuse. Neurol Sci 33:S169-S171

6. Buse DC, Manack A, Serrano D (2010) Sociodemographic and comorbidity profiles of chronic migraine and episodic migraine sufferers. J Neurol Neurosurg Psychiatry 81:428-432

7. Grazzi L, Bussone G (2011) What future for treatment of chronic migraine with medication overuse? Neurol Sci 32:S19-S22

8. Andrasik F, Grazzi L, Usai S et al (2010) Disability in chronic migraine with medication overuse: treatment effects through 5 years. Cephalalgia 30:610-614

9. Silberstein SD, Lipton RB, Sliwinski M (1996) Classification of daily and near-daily headaches. Neurology 47:871-875

10. D'Amico D, Mosconi P, Genco S et al (2001) The Migraine Disability Assessment (MIDAS) questionnaire: translation and reliability of the Italian version. Cephalalgia 21:947-952

11. Ustun TB, Chatterji S, Kostanjsek N et al (2010) Developing the world health organization disability assessment schedule 2.0. Bull World Health Organ 88:815-823

12. Beck AT, Steer RA, Brown GK (1996) Manual for the beck depression inventory-II. Psychological Corporation, San Antonio

13. Ashina S, Serrano D, Lipton RB et al (2012) Depression and risk of transformation of episodic to chronic migraine. J Headache Pain 13:615-624

14. Buse DC, Silberstein SD, Manack AN et al (2012) Psychiatric comorbidities of episodic and chronic migraine. J Neurol. doi: $10.1007 / \mathrm{s} 00415-012-6725-\mathrm{x}$ 